\title{
Resveratrol, a phytoestrogen found in red wine. A possible explanation for the conundrum of the 'French paradox'?
}

\begin{abstract}
Peter Kopp
Division of Endocrinology, Metabolism and Molecular Medicine, Northwestern University, Chicago, Illinois 60611, USA

(Correspondence should be addressed to P Kopp, Division of Endocrinology, Metabolism and Molecular Medicine, Northwestern University, Tarry 15, 303 Chicago Avenue, Chicago, Illinois 60611, USA)
\end{abstract}

Certain populations, e.g. the French and the Greek, suffer little heart disease despite a diet which is relatively high in fat. It has been proposed that regular consumption of red wine in moderate amounts may explain this phenomenon, which has been dubbed the 'French paradox' $(1,2)$. Resveratrol, a compound found in grapes and wine in significant amounts, was implicated in this beneficial action of red wine because of its ability to act as an antioxidant and an inhibitor of platelet aggregation $(1,3)$. In addition, resveratrol has been reported to have anticarcinogenic effects in mouse mammary cultures (4).

Gehm et al. have now reported on a fascinating new facet of this compound (5). On the basis of the structural similarity of resveratrol (trans-3, $4^{\prime}, 5$-trihydroxystilbene) to the synthetic estrogen diethylstilbestrol (4,4'-dihydroxy-trans- $\alpha, \beta$-diethylstilbene), these authors hypothesized that it might be a phytoestrogen. Given the known cardioprotective benefits of estrogens, this speculation appeared particularly appealing (6).

Using several experimental approaches, Gehm et al. found that resveratrol is indeed a phytoestrogen (5). Resveratrol was able to elicit a dose-dependent activation of a luciferase reporter gene under the control of an estrogen response element (ERE) linked to the minimal thymidine kinase promoter in MCF-7 cells, an estrogen receptor (ER)-containing human adenocarcinoma breast cell line. Half-maximal induction was observed at resveratrol levels of $5-10 \mu \mathrm{mol} / \mathrm{l}$. While less potent than estradiol, which displays maximal effects at doses of $0.1 \mathrm{nmol} / \mathrm{l}$, the maximal activation induced by resveratrol was several-fold higher than with estradiol. Suboptimal doses of resveratrol and estradiol were additive. At maximal activation by resveratrol, there was no further increase by estradiol. At maximal doses, resveratrol was as effective as estradiol in stimulating progesterone receptor gene expression in MCF-7 cells, but there was no superagonistic effect.

Deletion of the ERE from the reporter construct abolished the effect of resveratrol on luciferase activity. In addition, the estrogen antagonists tamoxifen and ICI 182,780 inhibited the induction by resveratrol, and resveratrol was unable to activate the reporter system in an ER-negative breast cancer cell line (MDA-MB-231), unless ERs were cotransfected. Resveratrol inhibited binding of ${ }^{125}$ I-labeled estradiol to the ER in competition binding studies using nuclear extracts of MCF-7 cells. Resveratrol was found to be a relatively weak ligand for the ER with an $\mathrm{IC}_{50}$ of $\sim 10 \mu \mathrm{mol} / \mathrm{l}$ at $0.1 \mathrm{nmol} / \mathrm{l}^{125} \mathrm{I}$ labeled estradiol. This concentration is similar to the levels needed to elicit other biological effects $(1,3)$.

In comparison with MCF-7 cells, the superagonistic effect of resveratrol was less pronounced in BG-1 ovarian carcinoma cells, suggesting a partial tissue specificity. In contrast to previous findings on a growth inhibitory effect of resveratrol in mouse mammary cultures (4), Gehm et al. observed that resveratrol induced proliferation of the estrogen-dependent human breast cancer cell line T47D (5). The growth-inducing effect of resveratrol was as pronounced as with estradiol, and it could be inhibited by addition of the antiestrogenic compound ICI 182,780.

Despite the concern that the estrogenic properties of resveratrol may have undesirable side effects because they result in the stimulation of human breast cancer cells, this study raises the interesting possibility that this phytoestrogen may contribute to the cardioprotective effects associated with red wine consumption. Although the risk for developing coronary heart disease is an equation with numerous variables, this speculation remains stimulating. It will be of interest to obtain more information on the relative resveratrol concentrations in wine. It is established that resveratrol concentrations are much higher in red wines compared with white wines. This may, at least in part, be explained by the fact that immediately after the grapes are pressed, the skins are removed in white wine production, while the grape skins are left with the freshly pressed red wine for variable periods of time in order to extract aromatic compounds. In a survey on the resveratrol content of red wines, Goldberg et al. reported a high variability in its concentrations (7). Differences in wine processing, type of grape and vintage, as well as climatic factors are thought to account for these variations. Resveratrol concentrations are generally higher in cooler climates because it is thought to play a role in the defence of the vine against fungal infections. 
Whether resveratrol is indeed implicated in the explanation of the 'French paradox' remains to be shown. Currently, there is no information on the serum concentration of resveratrol after red wine consumption, nor on its exact fate after ingestion. Besides these questions on the role of resveratrol as a dietary substance, one wonders what could explain the tissuespecificity of the superagonistic effect. Does resveratrol interact with both $\operatorname{ER} \alpha$ and $\operatorname{ER} \beta$, and what is the role of tissue-specific coactivators or corepressors? It would not be surprising if other phytoestrogens with similar features are characterized in the near future. Drinking a good glass of wine, we will certainly come up with a lot of additional questions on 'Château Hormone', a title given to a recent comment on this fascinating observation of Gehm et al. (8). Currently, we can only speculate how much and which vintage of 'Château Hormone' we should drink in order to benefit from the effects of resveratrol while avoiding the potentially detrimental actions of ethanol. Whatever the final verdict on resveratrol may be, let us finish here by wishing 'Santé!'.

\section{References}

1 Frankel EN, Parks EJ, Xu R, Schneeman BO, Davis PA \& German JB. Effect of $n-3$ fatty acid-rich fish oil supplementation on the oxidation of low density lipoproteins. Lipids 199429 233-236.

2 Goldberg DM, Hahn SE \& Parkes JG. Beyond alcohol: beverage consumption and cardiovascular mortality. Clinica Chimica Acta 1995237 155-187.

3 Bertelli AA, Giovannini L, Giannessi D, Migliori M \& Bernini W. Antiplatelet activity of synthetic and natural resveratrol in red wine. International Journal of Tissue Reactions 199517 1-3.

4 Jang M, Cai L, Udeani GO, Slowing KV, Thomas CF, Beecher CW et al. Cancer chemopreventive activity of resveratrol, a natural product derived from grapes. Science 1997275 218-220.

5 Gehm BD, McAndrews JM, Chien PY \& Jameson JL. Resveratrol, a polyphenolic compound found in grapes and wine, is an agonist for the estrogen receptor. Proceedings of the National Academy of Scienes of the USA 199794 14138-14143.

6 Lobo RA. Benefits and risks of estrogen replacement therapy. American Journal of Obstetrics and Gynecology $1995173982-989$.

7 Goldberg DM, Yan J, Ng E, Diamandis EP, Karumanchiri A, Soleas G et al. A global survey of trans-resveratrol concentrations in commercial wines. American Journal of Enology and Viticulture 199446 159-165.

8 Boyce N. Château hormone. New Scientist 199716. 